\title{
Poisoning in children 3: Common medicines
}

\section{Riordan, G Rylance, K Berry}

\section{A wide variety of medicines are ingested by children}

o $n$ this, the third of a series of articles on the management of poisoning, we deal with medicines commonly ingested by children.

\section{COUGH AND DECONGESTANT PREPARATIONS}

Proprietary preparations for the treatment of coughs, colds, and nasal congestion are frequently ingested accidentally. Most preparations (75\%) are made up of more than one active ingredient.

Sympathomimetic agents make up the largest group of ingredients. Their effects in overdose vary widely between agents. Individual susceptibility is also variable. Adverse effects are unusual and occur fairly soon after ingestion. Severe cases are associated with hypertension, reflex bradycardia, arrhythmias, convulsions, and coma. Treatment is supportive.

Paracetamol and aspirin make up the second biggest group of ingredients. Their presence should not be overlooked as measurement of blood concentration may be required, depending on the dose consumed.

Opioid analgesics are found in a number of preparations. Dextromethorphan is most frequently encountered. It has a low toxic potential and only produces side effects when ingested in considerable quantity. Codeine is also found in a number of preparations. It is more toxic in overdose.

Expectorants make up the final group of ingredients. These are mucosal irritants or stimulants. Their putative mechanism of action is to increase respiratory tract fluid, thereby facilitating its expulsion. Expectorants include guaiphenesin, ipecacuanha, ammonium salts, cineole, creosote, squill, and tolu. These agents may act as emetics in overdosage but because of their low dosage, toxicity is generally overshadowed by other ingredients.

The presence of several active ingredients in a single preparation may produce a confusing clinical picture and potentiate adverse effects. Poison centre advice should be sought if the maximum daily therapeutic dose of any preparation is exceeded.

\section{ORAL CONTRACEPTIVE PILLS}

Accidental ingestion of oral contraceptive pills rarely produces any toxic effect other than transient gastrointestinal upset on the following day. Parents should be advised that vaginal bleeding could occur, in girls of all ages, during the first few days following ingestion.

\section{ANTIBIOTICS}

Accidental overdose of most commonly prescribed antibiotics is associated with little in the way of adverse effects. Transient gastrointestinal disturbance may occur. No treatment is required.

\section{BENZODIAZEPINES}

These are most frequently encountered by children in the form of sedatives or anxiolytics prescribed for adults. In overdose they produce drowsiness, ataxia, hallucinations, confusion, and agitation. Respiratory depression, bradycardia, and hypotension can also occur.

Activated charcoal should be administered if presentation occurs within one hour of ingestion and conscious level is not impaired. Asymptomatic children should be observed for four hours. ${ }^{1}$

Symptomatic children require hospital admission. Treatment is largely supportive. The use of flumazenil, a competitive antagonist to benzodiazepines, is not generally indicated. Flumazenil can produce nausea, vomiting, flushing, and transient hypotension. Its use should be restricted to patients in whom adequate measures to protect the airway, support respiration, and maintain circulation have failed. Flumazenil may precipitate convulsions in patients on long term benzodiazepine treatment, for epilepsy.

\section{VITAMIN PREPARATIONS}

Vitamin preparations are generally of low toxicity in overdose, but they may contain iron and this may not be explicit from the product name.

\section{TOPICAL MEDICINAL PRODUCTS}

Most topical medicinal products are of low toxicity when ingested. Nausea, vomiting, and diarrhoea may occur. Exceptions include products with potentially toxic active ingredients, for example, salicylates in wart and callus treatments. Depilatory creams contain thioglycollate as an active ingredient. In small quantities they cause oropharyngeal irritation and burning if consumed. Larger amounts can cause corrosive injury or systemic toxicity.

\section{ASTHMA MEDICATION}

Salbutamol is the most common asthma medication to be accidentally ingested by children. In overdose salbutamol can induce tremor, tachycardia, agitation, metabolic acidosis, hyperglycaemia, and hypokalaemia. Symptomatic ingestion is uncommon and associated with large doses ( $1 \mathrm{mg} / \mathrm{kg}$ ).

Activated charcoal should be given if more than $1 \mathrm{mg} / \mathrm{kg}$ has been ingested. Asymptomatic children can be discharged at four hours post-ingestion. Symptoms are generally short lived and resolve spontaneously. ${ }^{2}$

Theophylline is far more toxic in overdose but is encountered infrequently. Symptoms include vomiting, tachycardia, tachypnoea, tremors, agitation, and convulsions. Metabolic disturbances include hyperglycaemia, hypokalaemia, hypophosphataemia, metabolic acidosis, and respiratory alkalosis.

Children who have consumed more than $15 \mathrm{mg} / \mathrm{kg}$ of theophylline require hospital admission and treatment with activated charcoal. Use of multiple doses of activated charcoal can enhance theophylline elimination ${ }^{3} ; 0.5 \mathrm{~g} / \mathrm{kg}$ should be administered on a four hourly basis.

Propanolol can be used to prevent the metabolic disturbances caused by theophylline overdose (most are mediated by direct sympathomimetic $\quad \beta_{2}$ stimulation). ${ }^{4}$ When these metabolic abnormalities are established it is far less efficacious. ${ }^{5}$ Hypokalaemia has been implicated in the induction of cardiac arrhythmias and convulsions which accompany theophylline poisoning. ${ }^{6}$ It should be corrected cautiously ${ }^{7}$ with intravenous supplementation. Hyperglycaemia is transient and does not require treatment.

Theophylline increases cardiac conduction velocity, increases catecholamine liberation, reduces coronary blood flow, and decreases the ventricular fibrillation threshold. It can produce both supraventricular and ventricular arrhythmias. Arrhythmias are concentration dependent and are exacerbated by hypoxia, hypercapnia, and acidosis. Children are less prone to theophylline induced arrhythmias than adults. ${ }^{5}$

Propanolol is the first line treatment for both supraventricular and ventricular arrhythmias. Esmolol is an alternative in asthmatic patients. ${ }^{5}$ Adenosine may also prove effective. ${ }^{1}$

\section{ANTIHISTAMINES}

Antihistamines cause central nervous system depression and anticholinergic 
side effects in overdose. Hypotension, muscle weakness, paradoxical central nervous system stimulation, and convulsions can also occur.

Antihistamine overdose can produce a variety of cardiac arrhythmias. For the majority of antihistamines this effect is mediated by myocardial sodium channel blockade-a quinidine like effect. Two of the newer non-sedating antihistamines, terfenadine and astemizole, cause delayed cardiac repolorisation by potassium channel blockade. This is associated with prolongation of the QT interval and may predispose to the development of ventricular tachyarrhythmias.

Children should be observed for a minimum of four hours following ingestion. Symptomatic children, and those who have ingested terfenadine, astemizole, or slow release preparations, even in the absence of symptoms, require admission. Activated charcoal should be considered up to four hours post-ingestion as gut motility is impaired. Treatment is otherwise largely supportive.

While the anticholinergic side effects of antihistamines are unpleasant they are not generally life threatening. The majority of clinically significant effects of antihistamine poisoning are not anticholinergic mediated. For this reason acetylcholinesterase inhibitors, such as physostigmine, are not recommended in antihistamine poisoning.

Hypotension should be treated with intravenous fluids. Where inotropes are considered necessary, adrenaline should be avoided as paradoxical hypotension can occur.

Convulsions and paradoxical excitement can be treated with a benzodiazepine. Phenothiazine based sedatives should be avoided because of their anticholinergic side effects.

Continuous ECG monitoring is advised for symptomatic children and those who have ingested terfenadine or astemizole. The management of arrhythmias in antihistamine poisoning can be difficult. Sinus tachycardia is the most frequently seen abnormality. This can usually be managed conservatively. A number of other arrhythmias can occur. Care must be taken in the choice of antiarrhythmic drugs. Sodium bicarbonate is the first drug of choice for QT prolongation with sodium channel blocking antihistamines. Class Ia and III antiarrhythmic drugs should be avoided in this situation. Magnesium sulphate may be considered for arrhythmias caused by terfenadine or astemizole.

\section{ANTIDEPRESSANTS}

Antidepressant medications include tricyclic compounds, monoamine oxidase inhibitors, and selective serotonin reuptake inhibitors.

Tricyclic compounds are prescribed as antidepressants for adult patients. Children may be prescribed tricyclic drugs for nocturnal enuresis, chronic pain disorders, or attention deficit disorder. In overdose tricyclics produce anticholinergic symptoms, drowsiness, ataxia, and agitation. Convulsions, central nervous system depression, and hypotension also occur.

Cardiac arrhythmias occur frequently and are the most common cause of death. The mechanism of cardiac toxicity is complex. Blockade of noradrenaline reuptake at the adrenergic synapse produces initial stimulation followed by $\alpha$ adrenergic blockade. Tricyclics also exert a quinidine like membrane stabilising effect. This causes delayed conduction and myocardial depression. The anticholinergic effects of tricyclics on the heart are not of major significance.

Activated charcoal should be administered if the maximum recommended daily dose has been exceeded.

A 12 lead ECG should be performed in all cases. The best indicator of risk for cardiac toxicity is a QRS duration of more than 0.1 seconds. ${ }^{8}$ Sinus tachycardia, QT or PR prolongation, and ventricular ectopics are all common.'

Asymptomatic children, with a normal ECG, should be observed for a minimum of six hours. Patients with any ECG abnormality should be monitored until resolution of the abnormality.

Compromising arrhythmias should be treated with an initial bolus of $1 \mathrm{ml} / \mathrm{kg}$ of $8.4 \%$ sodium bicarbonate. Arterial pH should subsequently be maintained between 7.45 and 7.55, using further doses of sodium bicarbonate as required. Arrhythmias unresponsive to sodium bicarbonate may be treated with phenytoin, atenolol, or propanolol. Class Ia antiarrhythmic drugs should be avoided. Life threatening arrhythmias may respond to cardioversion. Survival has been reported, in patients with tricyclic induced electromechanical dissociation, following several hours of external cardiac massage.

Hypotension unresponsive to intravenous fluids should be treated with sodium bicarbonate. Glucagon can also be used to treat refractory hypotension.

The large volume of distribution of tricyclic compounds means that they are not amenable to removal by extracorporeal means. ${ }^{10-14}$

Poisoning by monoamine oxidase inhibitors is uncommon following a decline in their use. In overdose they produce anticholinergic effects, excessive central nervous stimulation, convulsions, hyperpyrexia, and rhabdomyolysis. Activated charcoal should be administered following ingestion. Treatment is supportive. Dantrolene may be required to treat hyperpyrexia.

Selective serotonin reuptake inhibitor usage has increased in recent years because of their efficacy and more tolerable side effect profile when compared with tricyclic antidepressants. ${ }^{15}$ While these compounds are much safer in overdose than tricyclic antidepressants, ${ }^{16}{ }^{17}$ adverse effects have been documented. Adverse effects include vomiting, agitation, tremor, nystagmus, and drowsiness. Convulsions and arrhythmias $^{18}$ have also been reported.

A "serotonin syndrome" has been described. This most commonly occurs in adults receiving combinations of drugs, two or more of which inhibit the reuptake or metabolism of serotonin, or enhance its release. Examples of such drugs include selective serotonin reuptake inhibitors, tricyclic antidepressants, monoamine oxidase inhibitors, and amphetamines. Symptoms include autonomic dysfunction, hyperpyrexia, circulatory collapse, convulsions, and rhabdomyolysis. ${ }^{19}$ Reports suggest that this constellation of symptoms may also occur following the ingestion of a single agent. ${ }^{20}{ }^{21}$ Treatment is supportive.

\section{IRON}

Early features of iron poisoning include vomiting, diarrhoea, and abdominal pain. Direct mucosal irritation by adherent tablets can cause gastrointestinal haemorrhage. These effects usually settle within six hours.

Absorbed iron is rapidly cleared from the circulation by cellular uptake. High concentrations of intracellular iron disrupt mitochondrial function and result in cell death. Signs of multiorgan failure present at 12-48 hours post-ingestion. The liver is particularly prone to damage and symptoms of fulminant hepatic failure predominate.

A careful history must be taken as to the iron preparation consumed and the maximum quantity taken. Different iron salts contain differing quantities of elemental iron: for example, $200 \mathrm{mg}$ of ferrous sulphate contains $65 \mathrm{mg}$ of elemental iron; $300 \mathrm{mg}$ of ferrous gluconate contains $35 \mathrm{mg}$.

Asymptomatic children, with a definite history of consuming less than 30 $\mathrm{mg} / \mathrm{kg}$ of elemental iron, do not require further investigation. Activated charcoal does not bind iron.

Children who may have consumed more than $30 \mathrm{mg} / \mathrm{kg}$ require hospital admission and further investigation. An abdominal $x$ ray examination should be performed. If undissolved iron tablets are visible on the abdominal film, whole bowel irrigation should be undertaken. Irrigation should continue until abdominal films are clear of undissolved tablets. ${ }^{22}$ In patients with tablets confined to the stomach, repeated gastric lavage or endoscopic removal are alternative strategies. The absence of iron tablets on abdominal $x$ ray does not preclude the presence of a significant ingestion. Vitamin tablets containing iron 
Table 1 Products of low toxicity

Cold sore treatment

Ear and eye drops

Hormone replacement tablets

Nasal drops

Steroids

Note: Some of the products listed present a significant choking hazard that should not be overlooked.

may not be demonstrable radiologically. If large quantities have been consumed, whole bowel irrigation should be undertaken.

In asymptomatic patients, a serum iron level should be determined at four hours post-ingestion. This level should be repeated at eight hours if a sustained release preparation has been consumed. Patients with a serum iron level less than $55 \mu \mathrm{mol} /$ /require no further treatment, and, provided gastric decontamination is not required, can be discharged. Patients with a serum iron level between 55 and $90 \mu \mathrm{mol} / \mathrm{l}$ require observation for at least 24 hours. If they remain asymptomatic, no specific treatment is indicated.

Patients with a serum iron level greater than $90 \mu \mathrm{mol} / \mathrm{l}$ should receive treatment with intravenous desferrioxamine.

Patients with symptoms suggestive of multiorgan failure require intensive support and immediate treatment with intravenous desferrioxamine. Expert advice should be sought, as a higher total daily dose may prove beneficial. As desferrioxamine interferes with serum iron estimation, blood levels should be taken prior to commencing treatment. Treatment, however, should not be delayed whilst results are awaited

Desferrioxamine chelates free iron. The chelate complex is excreted in the urine, giving it a pink-brown tinge (vinrosé). Should renal function be significantly impaired this complex can also be removed by haemodialysis.

Desferrioxamine treatment should be continued until symptoms have abated and urine discolouration clears. Specialist advice should be sought if therapy seems likely to exceed a period of 24 hours.
Gastrointestinal stricture formation, caused by the corrosive effects of iron, can occur as a late complication of iron poisoning.

\section{INGESTIONS OF LOW TOXICITY}

A wide range of medicines are accidentally ingested by children. Many are of very low toxicity (see table 1 ).

\section{SEEKING FURTHER ADVICE}

Specific, expert advice on all aspects of poisoning is available to medical professionals in the United Kingdom via the National Poisons Information Service (NPIS). The regional centres that make up this service have recently introduced a single national enquiry number: 08706006266.

A wide range of easily accessible and highly practical advice is available through the NPIS website. ${ }^{23}$ This free service is restricted to medical professionals. On line registration is available at http://www.spib.axl.co.uk/toxbase/.

\section{Arch Dis Child 2002;87:400-402}

\section{Authors' affiliations}

M F Riordan, Department of Pediatrics, Yale University Medical School, USA

G W Rylance, Department of General

Paediatrics, Royal Victoria Infirmary, Newcastle upon Tyne, UK

K Berry, Accident and Emergency Department, Birmingham Children's Hospital, Birmingham, UK

Correspondence to: Dr K Berry, Accident and Emergency Department, Birmingham Children's Hospital, Steelhouse Lane, Birmingham B4 6NH, UK;

kathleen.berry@bhamchildrens.wmids.nhs.uk

\section{REFERENCES}

1 Bates N, Edwards N, Roper J, et al. Paediatric toxicology: handbook of poisoning in children. London: Macmillan Reference Limited, 1997

2 Wiley JF, Spiller HA, Krenzelok EP, et al. Unintentional albuterol ingestion in children. Pediatr Emerg Care 1994:10:193-6.

3 Vale JA, Krenzelok EP, Barceloux GD Position statement and practice guidelines on the use of multi-dose activated charcoal in the treatment of acute poisoning. American Academy of Clinical Toxicology; European Association of Poisons Centres and Clinical Toxicologists. J Toxicol Clin Toxicol 1999;37 731-51.
4 Kearney TE, Manoguerra AS, Curtis GP et al. Theophylline toxicity and the beta-adrenergic system. Ann Intern Med 1985;102:766-9.

5 Gaudreault P, Guay J. Theophylline poisoning. Pharmacological considerations and clinical management. Med Toxicol 1986:1:169-91

6 Boldy DAR, Buckley BM, Vale JA. Advances in the treatment of poisoning I. J Clin Hosp Pharm 1984:(9): 1 47-60.

7 D'Angio R, Sabatelli F. Management D'Angio R, Sabatelli F. Management
considerations in treating metabolic considerations in treating metabolic
abnormalities associated with theophylline overdose. Arch Intern Med 1987; 147: 1837-8.

8 Boehnert MT, Lovejoy FN. Value of the QRS duration versus the serum drug level in predicting seizures and ventricular arrhythmias after acute overdose of tricyclic arrhythmias after acute overdose antidepressants. N

9 Commerford PJ, Lloyd EA. Arrhythmias in patients with drug toxicity, electrolyte, and endocrine disturbances. Med Clin North Am 1984;68:1051-79.

10 Pond SM. Extracorporeal techniques in the treatment of poisoned patients. Med J Aust 1991;154:617-22.

11 Bismuth C. Biological valuation of extra-corporeal techniques in acute poisoning. Acta Clin Belg Suppl 1990;13:20-8.

12 Heath A, Wickstrom I, Martensson E, et al. Treatment of antidepressant poisoning with resin hemoperfusion. Hum Toxicol 1982;1:361-71.

13 Henry JA. Can tricyclic antidepressants be removed by haemoperfusion? [editorial]. Hum Toxicol 1982;1:359-60.

14 Haapanen EJ. Hemoperfusion in acute intoxication. Clinical experience with 48 cases. Acta Med Scand Suppl 1982;668:76-81.

15 Catalano G, Cooper DS, Catalano MC, et al. Pediatric sertraline overdose. Clin Neuropharmacol 1998:21:59-61.

16 Personne $M$, Sjoberg G, Persson $\mathrm{H}$. Citalopram overdose-review of cases treated in Swedish hospitals [published erratum appears in J Toxicol Clin Toxicol 1997;35:577]. J Toxicol Clin Toxico 1997; 35:237-40.

17 Borys DJ, Setzer SC, Ling L, et al. Acute fluoxetine overdose: a report of 234 cases. Am J Emerg Med 1992;10:115-20.

18 Graudins A, Vossler C, Wang R. Fluoxetine-induced cardiotoxicity with response to bicarbonate therapy. Am J Emerg Med 1997; 15:501-3.

19 Francois B, Marquet P, Desachy A, et al. Serotonin syndrome due to an overdose of moclobemide and clomipramine. A potentially life-threatening association. Intensive Care Med 1997;23:122-4.

20 Gill M, LoVecchio F, Selden B. Serotonin syndrome in a child after a single dose of fluvoxamine. Ann Emerg Med 1999;33:457-9.

21 Mueller PD, Korey WS. Death by "ecstasy": the serotonin syndrome? Ann Emerg Med 1998;32(3 pt 1):377-80.

22 Tenenbein M. Position statement: whole bowel irrigation. American Academy of Clinical Toxicology; European Association of Poisons Centres and Clinical Toxicologists [review]. J Toxicol Clin Toxicol 1997;35:753-62.

23 Good AM, Bateman DN. TOXBASE on the Internet. J Accid Emerg Med 1999;16:399. 